\title{
2D systems based robust iterative learning control using noncausal finite-time interval data
}

\author{
Błażej Cichy ${ }^{\mathrm{a}, *}$, Krzysztof Gałkowski ${ }^{\mathrm{a}}$, Eric Rogers ${ }^{\mathrm{b}}$ \\ ${ }^{a}$ Institute of Control and Computation Engineering, University of Zielona Góra, ul. Podgórna 50, 65-246 Zielona Góra, Poland \\ ${ }^{\mathrm{b}}$ Electronics and Computer Science, University of Southampton, Southampton SO17 1BJ, UK
}

\section{A R T I C L E I N F O}

\section{Article history:}

Received 3 April 2013

Received in revised form

18 July 2013

Accepted 6 November 2013

\section{Keywords:}

Iterative learning control

Robust control

2D systems

Noncausal data

\begin{abstract}
A B S T R A C T
This paper uses a 2D system setting in the form of repetitive process stability theory to design an iterative learning control law that is robust against model uncertainty. In iterative learning control the same finite duration operation, known as a trial over the trial length, is performed over and over again with resetting to the starting location once each is complete, or a stoppage at the end of the current trial before the next one begins. The basic idea of this form of control is to use information from the previous trial, or a finite number thereof, to compute the control input for the next trial. At any instant on the current trial, data from the complete previous trial is available and hence noncausal information in the trial length indeterminate can be used. This paper also shows how the new 2D system based design algorithms provide a setting for the effective deployment of such information.
\end{abstract}

(C) 2013 Elsevier B.V. All rights reserved.

\section{Introduction}

Many systems complete the same finite duration operation over and over again, where each execution is known as a trial and the duration the trial length. The exact sequence is that on completion of each trial, the system resets to the starting location and the next one begins. A common application is replicated by a robot undertaking a pick and place task over and over again, i.e., collect an object from a specified location, transfer it over a finite duration, deposit it at a fixed location or onto a moving conveyor, return to the starting location and then repeat this sequence of operations. In this paper, the notation used is $y_{k}(p), 0 \leq p \leq \alpha-1, k \geq 0$, where $y$ is the vector or scalar variable under consideration, $\alpha<\infty$ is the number of samples along the trial length and $k$ is the trial number. Also if $y_{\text {ref }}(p), 0 \leq p \leq \alpha-1$, is a given reference trajectory for the output, which is assumed to be a member of the signal space chosen for the output of the controlled system, $e_{k}(p)=$ $y_{\text {ref }}(p)-y_{k}(p)$ is the error on trial $k$ and in iterative learning control (ILC) the novel feature is the use of the previous trial error in the computation of the control input applied on the next trial, with a generalization to higher-order ILC where the errors from a finite number $l>1$ of previous trials are used.

\footnotetext{
This work is partially supported by the National Science Centre in Poland, grant No. 2011/01/B/ST7/00475.

* Corresponding author. Tel.: +48 683284733 .

E-mail addresses: b.cichy@issi.uz.zgora.pl (B. Cichy),

k.galkowski@issi.uz.zgora.pl (K. Gałkowski), etar@ecs.soton.ac.uk (E. Rogers).
}

Since its inception, widely credited to [1], ILC has seen extensive developments from theory through to experimental benchmarking and actual applications. The survey papers [2,3] are starting points for the literature and these together with subsequent publications show a wide range of applications from industrial robotics to residual vibration suppression, microelectronics fabrication, process control and recently a technology transfer to healthcare in the form of robotic-assisted upper limb stroke rehabilitation. In this latter application [4] the patient makes repeated attempts to follow a reference trajectory replicating daily living tasks, such as reaching out to a cup across a table top, assisted by a robot and with electrical stimulation applied to the relevant muscles. At the end of each trial, the patient's arm is returned to the starting location and ILC used to compute the electrical stimulation to be applied on the next trial based on the previous trial error. If the patient is improving with increasing trial number then his/her voluntary effort should increase and the applied stimulation decrease and this has been confirmed in clinical trials.

In ILC, all previous trial data are available before the next trial begins and hence there is not a requirement that only causal in $p$ previous trial data is used in the computation of the current trial input, i.e., the control applied at $p \in[0, \alpha]$ on trial $k$ can use previous trial data at $p=p+1, \ldots, \alpha$. The ability to use the noncausal information is a novel feature of ILC and many successful implementations use the special case of phase-lead where a term in $e_{k}(p+\lambda), \lambda>0$ is used to form the control input $u_{k+1}(p)$ on the next trial, where $\lambda=1$ is common. In such cases, one alternative to consider is the inclusion of a weighted sum of previous trial error phase-lead terms or a weighted sum of such terms and ILC 
lag terms $e_{k}(p-\beta), \beta>0$. The aim of this paper is to provide the basis of ILC design where the use of such information can be evaluated as a necessary step toward implementation.

If the along the trial dynamics are discrete then one setting for ILC design is, since the trial length is finite, to define supervectors for the variables. For example, let the system be singleinput single-output for the ease of presentation with a natural extension to the vector case. Then the error super-vector is $E_{k}=$ $\left[\begin{array}{llll}e_{k}(0) & e_{k}(1) & \ldots & e_{k}(\alpha-1)\end{array}\right]^{T}$ and the ILC error dynamics can hence be written as a system of linear difference equations in $k$ of the form $E_{k+1}=H E_{k}$.

Due to the finite trial length, error convergence in $k$ can occur even if the system has an unstable state matrix. The solution via lifting design is to first design a stabilizing feedback control law and then apply ILC to the resulting controlled system. Also for robust control based on norm bounded or polytopic uncertainty, the entries in the matrix $H$ will contain products of the matrices describing the uncertainty and this makes the analysis significantly more involved. Moreover, robustness analysis in the frequency domain is approximated as ILC controllers operate on a finite time interval, the trial length. Use of the Fourier transform on the infinite time interval will give a linear time-invariant control law but over the finite trial length errors may result in the initial part of the transient response; an example to support this fact is given in [5]. A robust $H_{\infty}$ based ILC design with noncausal finite trial length is also given in [5] but is somewhat involved and choosing exactly what noncausal data to include is also lacking somewhat in transparency.

An alternative to the lifted model analysis is to exploit the natural 2D system structure of ILC where one direction of information is from trial-to-trial, indexed by the subscript $k$, and the other along a trial, indexed by $p$. The first results on 2D system based ILC analysis is credited to [6] where a Roesser state-space model was used. Repetitive processes [7] are another class of 2D systems where information propagation in $p$ is over a finite duration and therefore a more obvious setting for analysis. The previous work on ILC laws designed using repetitive process control theory with experimental verification includes $[8,9]$. This setting also extends to differential dynamics and for robust control studies avoids products of matrices describing the uncertainty assumed. This paper will show that the repetitive process setting enables control law design where a weighted sum of noncausal and/or causal in $p$ is used and hence, for a given example, different combinations of previous trial terms can be considered in the search for a design that meets the performance specifications, with an extension to robust control. The relative merits of this design method are also discussed.

Throughout this paper, the null and identity matrices with the required dimensions are denoted by 0 and $I$ respectively. Also $M \succ 0(\prec 0)$ denotes a real symmetric positive (negative) definite matrix and $X \preceq Y$ is used to represent the case when $X-Y$ is a negative semi-definite matrix. Finally, $\operatorname{diag}\{\cdots\}$ denotes a block diagonal matrix.

\section{System representation and design}

\subsection{Discrete linear repetitive processes}

Discrete linear repetitive processes evolve over the subset of the positive quadrant in the $2 \mathrm{D}$ plane defined by $\{(p, k): 0 \leq p \leq$ $\alpha-1, k \geq 0\}$, and the basic state-space model for their dynamics has the following form [7]:

$\tilde{x}_{k+1}(p+1)=A \tilde{x}_{k+1}(p)+B \tilde{u}_{k+1}(p)+B_{0} \tilde{y}_{k}(p)$,

$\tilde{y}_{k+1}(p)=C \tilde{x}_{k+1}(p)+D \tilde{u}_{k+1}(p)+D_{0} \tilde{y}_{k}(p)$.

In this model on pass $k, \tilde{x}_{k}(p) \in \mathbb{R}^{n}$ is the state vector, $\tilde{y}_{k}(p) \in \mathbb{R}^{m}$ is the pass profile vector, and $\tilde{u}_{k}(p) \in \mathbb{R}^{r}$ is the vector of control inputs. The simplest form of boundary conditions is $\tilde{x}_{k+1}(0)=$ $d_{k+1}, k \geq 0$, where the $n \times 1$ vector $d_{k+1}$ has known constant entries and $\tilde{y}_{0}(p)=f(p)$, where $f(p)$ is an $m \times 1$ vector whose entries are known functions of $p$.

Repetitive process has their origins in the coal mining industry where in longwall coal cutting the machine rests on the previous pass profile, which is the height of the stone/coal interface above some datum line, during the production of the current pass profile. It is therefore unrealistic to assume that at instance $p$ on the current pass the only previous pass profile contribution comes from the same instance on the previous pass. An alternative model is

$$
\begin{aligned}
& \tilde{x}_{k+1}(p+1)=A \tilde{x}_{k+1}(p)+B \tilde{u}_{k+1}(p)+\sum_{i=-w_{l}}^{w_{h}} B_{i} \tilde{y}_{k}(p+i), \\
& \tilde{y}_{k+1}(p)=C \tilde{x}_{k+1}(p)+D \tilde{u}_{k+1}(p)+\sum_{i=-w_{l}}^{w_{h}} D_{i} \tilde{y}_{k}(p+i),
\end{aligned}
$$

where $w_{l}$ and $w_{h}$ are positive integers and the boundary conditions applied in this paper are those for (1), with the additional assumption that

$\tilde{y}_{k}(p)=0, \quad p \in\left\{-w_{l}, \ldots,-1\right\} \cup\left\{\alpha, \ldots, \alpha+w_{h}-1\right\}$.

Setting $w_{l}=0$ and $w_{h}=0$ recovers the previous model. In this alternative model on pass $k$ and instance $p$ the previous pass profile contribution is modeled as a linear sum of those at $0 \leq p-w_{l} \leq$ $p \leq p+w_{h} \leq \alpha-1$. The resulting model structure has no $2 \mathrm{D}$ discrete linear system state-space model interpretation, such as the Roesser model [10]. This paper applies the stability theory for this repetitive process model to ILC design.

\subsection{Iterative learning control (ILC)}

The systems considered are assumed to be modeled by linear time-invariant dynamics in the discrete domain with state-space model triple $\left\{A_{d}, B_{d}, C_{d}\right\}$. In ILC analysis, notation for the trial dependence is required. In this paper the subscript $k$ is used, giving the following description:

$x_{k}(p+1)=A_{d} x_{k}(p)+B_{d} u_{k}(p), x_{k+1}(0)=d_{k+1}$

$y_{k}(p)=C_{d} x_{k}(p), \quad 0 \leq p \leq \alpha-1$,

where $k \geq 0$ is the trial number, $\alpha<\infty$ is the number of samples along the trial, $x_{k}(p) \in \mathbb{R}^{n}$ is the state vector, $y_{k}(p) \in \mathbb{R}^{m}$ is the output vector, $u_{k}(p) \in \mathbb{R}^{l}$ is the control input vector and the entries in $d_{k+1}$ are known constants.

Let $y_{r e f}(p) \in \mathbb{R}^{m}$ denote the reference vector, which is assumed to be a member of the signal space chosen for the output of the controlled system, and hence the error on trial $k$ is

$e_{k}(p)=y_{r e f}(p)-y_{k}(p)$.

A commonly used ILC strategy constructs the current trial input as sum of that used on the previous one plus a corrective term, i.e.,

$u_{k+1}(p)=u_{k}(p)+\Delta u_{k+1}(p), \quad k \geq 0$,

where $\Delta u_{k+1}(p)$ denotes the correction term.

Introduce, for analysis purposes only, the vector

$\eta_{k+1}(p+1)=x_{k+1}(p)-x_{k}(p)$.

Suppose also that in the ILC law (6)

$\Delta u_{k+1}(p)=K_{1} \eta_{k+1}(p+1)+K_{2} e_{k}(p+1)$, 
where $K_{1}$ and $K_{2}$ are matrices to be designed. Then the application of this control law to (4) gives the controlled dynamics state-space model

$\eta_{k+1}(p+1)=\widehat{A} \eta_{k+1}(p)+\widehat{B}_{0} e_{k}(p)$,

$e_{k+1}(p)=\widehat{C} \eta_{k+1}(p)+\widehat{D}_{0} e_{k}(p)$

where

$\widehat{A}=A_{d}+B_{d} K_{1}, \quad \widehat{B}_{0}=B_{d} K_{2}$,

$\widehat{C}=-C_{d}\left(A_{d}+B_{d} K_{1}\right), \quad \widehat{D}_{0}=I-C_{d} B_{d} K_{2}$.

The state-space model $(8)$ is that of a discrete linear repetitive process of the form (1) with the input terms deleted and pass output and state vectors $e_{k+1}$ and $\eta_{k+1}$, respectively, once the boundary conditions are specified, i.e., the pass state initial vector $\eta_{k}(0), k \geq 0$, and the initial pass profile $e_{0}(p), 0 \leq p \leq \alpha-1$. As this paper uses the repetitive process setting for analysis and design, the word pass will be used instead of trial from this point onward. Without loss of generality, it is assumed that $\eta_{k+1}(0)=$ $0, k \geq 0$.

In [8], LMI based formulas for the computation of the control law matrices $K_{1}$ and $K_{2}$ are developed and for implementation the control law can be written using (6) and (7) as

$$
\begin{aligned}
u_{k}(p)= & u_{k-1}(p)+K_{1}\left(x_{k}(p)-x_{k-1}(p)\right) \\
& +K_{2}\left(y_{\text {ref }}(p+1)-y_{k-1}(p+1)\right)
\end{aligned}
$$

and the term $K_{2}\left(y_{\text {ref }}(p+1)-y_{k-1}(p+1)\right)$ is phase-lead ILC, i.e., noncausal in $p$. This control law also requires all entries in the state vector to be available for measurement and [9] produces an alternative where the difference in the state vectors on two successive trials is replaced by the difference in the trial output vectors on the same two trials.

The use of noncausal information is a major novel feature of ILC where many of the similar model designs that have been implemented use a form of phase-lead. As with all other simple structure control laws, there will be cases where such action will not be able to meet the performance specifications. In such cases, one alternative to consider is the inclusion of a weighted sum of previous trial error phase-lead terms or a weighted sum of such terms and ILC lag terms $e_{k}(p-\beta), \beta>0$. This paper develops an LMI based design for such a law based on the repetitive process state-space model (2) with an extension to robust control.

Consider the case when (7) is replaced by

$\Delta u_{k+1}(p)=K \eta_{k+1}(p+1)+\sum_{i=-w_{l}}^{w_{h}} K_{i} e_{k}(p+i+1)$.

Then, following the analysis for the application of the control law (11), the controlled dynamics state-space model is

$\eta_{k+1}(p+1)=\widehat{A} \eta_{k+1}(p)+\widehat{B}_{0} e_{k}(p)+\sum_{\substack{i=-w_{l} \\ i \neq 0}}^{w_{h}} \widehat{B}_{i} e_{k}(p-i)$,

$e_{k+1}(p)=\widehat{C} \eta_{k+1}(p)+\widehat{D}_{0} e_{k}(p)-\sum_{\substack{i=-w_{l} \\ i \neq 0}}^{w_{h}} \widehat{D}_{i} e_{k}(p-i)$,

which is of the form of (2) with

$\widehat{A}=A_{d}+B_{d} K, \quad \widehat{B}_{0}=B_{d} K_{0}$,

$\widehat{C}=-C_{d}\left(A_{d}+B_{d} K\right), \quad \widehat{D}_{0}=I-C_{d} B_{d} K_{0}$,

$\widehat{B}_{i}=B_{d} K_{i}, \quad \widehat{D}_{i}=-C_{d} B_{d} K_{i}, \quad i=-w_{l}, \ldots, w_{h}, i \neq 0$

and the assumption that

$e_{k}(p)=0, \quad p \in\left\{-w_{l}, \ldots,-1\right\} \cup\left\{\alpha, \ldots, \alpha+w_{h}-1\right\}$.
The stability problem for linear repetitive processes is that the sequence of pass profiles can contain oscillations that increase in amplitude from pass-to-pass ( $k$ variable) and the stability theory for linear constant pass length examples is defined using an abstract model of the dynamics in a Banach space setting [7]. In this model, the pass-to-pass updating is of the form $y_{k+1}=L_{\alpha} y_{k}$ where $y_{k} \in E_{\alpha}$ is a Banach space and $L_{\alpha}$ is a bounded linear operator mapping $E_{\alpha}$ into itself. The property of stability along the pass requires the existence of finite real scalars $M_{\infty}>0$ and $\lambda_{\infty} \in$ $(0,1)$ such that $\left\|L_{\alpha}^{k}\right\| \leq M_{\infty} \lambda_{\infty}^{k}$. For the autonomous case, i.e., the only contribution to the current trial pass profile is the previous one, this condition will ensure that the sequence of pass profiles produced will converge in $k$ to zero. In the ILC application, the pass profile on any pass is the error and hence the direct application of repetitive process stability theory to ILC error convergence.

One way of characterizing the stability of discrete linear repetitive processes that also leads to control law design algorithms is to use a Lyapunov function that is the sum of quadratic terms in the current pass state vector and the previous pass profile respectively. Stability along the pass then holds if the increment of this function, again the sum of that for each term, is negative definite. Using this approach gives the following result with formulas for the matrices in the ILC law (11).

Theorem 1. The repetitive process representing the ILC scheme of (12) is stable along the pass if there exist matrices $P \succ 0, N, Q_{i} \succ$ 0 , and $N_{i}, i=-w_{l}, \ldots, w_{h}$, such that the following LMI is feasible:

$\left[\begin{array}{cc}-\mathcal{P} & (\bar{A} \mathcal{P}+\bar{B} \mathcal{N})^{T} \\ \bar{A} \mathcal{P}+\bar{B} \mathcal{N} & -\mathcal{P}\end{array}\right] \prec 0$,

where

$\bar{A}=\left[\begin{array}{cccccccc}A_{d} & 0 & \cdots & 0 & I & 0 & \cdots & 0 \\ -C_{d} A_{d} & 0 & \cdots & 0 & I & 0 & \cdots & 0 \\ \vdots & \vdots & \ddots & \vdots & \vdots & \vdots & \ddots & \vdots \\ -C_{d} A_{d} & 0 & \cdots & 0 & I & 0 & \cdots & 0\end{array}\right]$,

$\bar{B}=\left[\begin{array}{ccc}B_{d} & \cdots & B_{d} \\ -C_{d} B_{d} & \cdots & -C_{d} B_{d} \\ \vdots & \ddots & \vdots \\ -C_{d} B_{d} & \cdots & -C_{d} B_{d}\end{array}\right]$,

and

$\mathcal{P}=\operatorname{diag}\left\{P, Q_{-w_{l}}, \ldots, Q_{0}, \ldots, Q_{w_{h}}\right\}$,

$\mathcal{N}=\operatorname{diag}\left\{N, N_{-w_{l}}, \ldots, N_{0}, \ldots, N_{w_{h}}\right\}$.

If the LMI of (15) holds, stabilizing matrices in the control law (11) are given by

$K=N P^{-1}$,

$K_{i}=N_{i} Q_{i}^{-1}, \quad i=-w_{l}, \ldots, w_{h}$.

Proof. Introduce the Lyapunov function; see also [11],

$V(k, p)=V_{1}(k, p)+V_{2}(k, p)$,

where

$V_{1}(k, p)=\sum_{i=-w_{l}}^{w_{h}} y_{k}^{T}(p+i) Q_{i} y_{k}(p+i)$

and

$V_{2}(k, p)=x_{k}^{T}(p) P x_{k}(p)$, 
with $P \succ 0$ and $Q_{i} \succ 0, i=-w_{l}, \ldots, w_{h}$. The term $V_{1}(k, p)$ captures the pass-to-pass energy change and $V_{2}(k, p)$ the change in energy along a pass. Moreover, the associated increment is

$$
\begin{aligned}
\Delta V(k, p)= & y_{k+1}^{T}(p)\left(\sum_{i=-w_{l}}^{w_{h}} Q_{i}\right) y_{k+1}(p) \\
& +x_{k+1}^{T}(p+1) P x_{k+1}(p+1) \\
& -\sum_{i=-w_{l}}^{w_{h}} y_{k}^{T}(p+i) Q_{i} y_{k}(p+i)-x_{k+1}^{T}(p) P x_{k+1}(p) .
\end{aligned}
$$

Summing from $p=0$ to $p=\alpha-1$ gives the global Lyapunov function

$V(k)=\sum_{p=0}^{\alpha-1} V(k, p)$,

with associated increment

$\Delta V(k)=\sum_{p=0}^{\alpha-1} \Delta V(k, p)$.

Following the steps in the proof of the Lyapunov function characterization for a process described by (1) in [7] gives that (2) is stable along the pass if

$\Delta V(k)<0$.

The proof is complete since (15) is a direct consequence of (25) on routine application of a congruence transform and use of Schur's complement formula.

The control law matrices $K_{i}, i=-w_{l}, \ldots, w_{h}$, in this last result can be parameterized using the optimization procedure

$\operatorname{maximize}\left(\sum_{i=-w_{l}}^{w_{h}} \operatorname{tr}\left(Q_{i}\right)+\sum_{i=-w_{l}}^{w_{h}} \operatorname{se}\left(N_{i}\right)\right)$

subject to:

$\left[\begin{array}{cc}-\mathcal{P} & (\bar{A} \mathcal{P}+\bar{B} \mathcal{N})^{T} \\ \bar{A} \mathcal{P}+\bar{B} \mathcal{N} & -\mathcal{P}\end{array}\right]<0$,

$\zeta \cdot \operatorname{tr}\left(Q_{i}\right) \leq \operatorname{se}\left(N_{i}\right), \quad i=-w_{l}, \ldots, w_{h}$,

where $\operatorname{tr}(\cdot)$ denotes the trace of a square matrix, se $(\cdot)$ is the sum of all elements in a matrix and the value of $\zeta$ in (26c) is chosen for a particular case by trial and error to guarantee the fastest pass-to-pass error convergence compatible with other design specifications.

\section{Robust control}

In this section the case considered is when the model matrices $A_{d}, B_{d}$ of (4) belong to a convex bounded (polytope type) uncertain domain $\mathscr{D}$, where any uncertain matrix can be written as a convex combination of its vertices

$$
\begin{aligned}
\mathscr{D}= & \left\{\left[A_{d}(\xi(k, p)), B_{d}(\xi(k, p))\right]:\left[A_{d}(\xi(k, p)), B_{d}(\xi(k, p))\right]\right. \\
= & \sum_{v=1}^{n_{v}} \xi_{v}(k, p)\left[A_{d v}, B_{d v}\right] ; \sum_{v=1}^{n_{v}} \xi_{v}(k, p)=1 ; \\
& \left.\xi_{v}(k, p) \geq 0 ; k \geq 0 ; 0 \leq p \leq \alpha-1\right\},
\end{aligned}
$$

where $A_{d v}, B_{d v}$ are the corresponding matrix vertices and $n_{v}$ denotes the number of them. This description allows variability of the model parameters in both $k$ (pass-to-pass) and along the pass $(p)$ within the assumed polytope. The matrix $C_{d}$ describing the contribution of the current pass state vector to the current pass profile, i.e. measured outputs, is assumed not to be uncertain since otherwise the repetitive process matrices do not form a convex set.

The state-space model of the repetitive process dynamics with the polytopic uncertainty (27) is obtained by replacing (4) with

$x_{k}(p+1)=A_{d}(\xi(k, p)) x_{k}(p)+B_{d}(\xi(k, p)) u_{k}(p)$,

$y_{k}(p)=C_{d} x_{k}(p)$,

and the robust ILC dynamics are described by

$$
\begin{aligned}
\eta_{k+1}(p+1)= & \widehat{A}(\xi(k, p)) \eta_{k+1}(p)+\widehat{B}_{0}(\xi(k, p)) e_{k}(p) \\
& +\sum_{\substack{i=-w_{l} \\
i \neq 0}}^{w_{h}} \widehat{B}_{i}(\xi(k, p)) e_{k}(p-i), \\
e_{k+1}(p)=\widehat{C}(\xi(k, p)) \eta_{k+1}(p)+\widehat{D}_{0}(\xi(k, p)) e_{k}(p) & -\sum_{\substack{i=-w_{l} \\
i \neq 0}}^{w_{h}} \widehat{D}_{i}(\xi(k, p)) e_{k}(p-i),
\end{aligned}
$$

where

$\widehat{A}(\xi(k, p))=A_{d}(\xi(k, p))+B_{d}(\xi(k, p)) K$,

$\widehat{B}_{0}(\xi(k, p))=B_{d}(\xi(k, p)) K_{0}$,

$\widehat{C}(\xi(k, p))=-C_{d}\left(A_{d}(\xi(k, p))+B_{d}(\xi(k, p)) K\right)$,

$\widehat{D}_{0}(\xi(k, p))=I-C_{d} B_{d}(\xi(k, p)) K_{0}$,

$\widehat{B}_{i}(\xi(k, p))=B_{d}(\xi(k, p)) K_{i}$

$\widehat{D}_{i}(\xi(k, p))=-C_{d} B_{d}(\xi(k, p)) K_{i}, \quad i=-w_{l}, \ldots, w_{h}, \quad i \neq 0$

and

$A_{d}(\xi(k, p))=\sum_{v=1}^{n_{v}} \xi_{v}(k, p) A_{d v}$,
$B_{d}(\xi(k, p))=\sum_{v=1}^{n_{v}} \xi_{v}(k, p) B_{d v}$.

The following result enables robust ILC design.

Theorem 2. The repetitive process representing the uncertain ILC dynamics of (29) is stable along the pass if there exist matrices $P>$ $0, N, Q_{i}>0$, and $N_{i}, i=-w_{l}, \ldots, w_{h}$, such that the following $L M I$ is feasible:

$\left[\begin{array}{cc}-\mathcal{P} & \left(\bar{A}_{v} \mathcal{P}+\bar{B}_{v} \mathcal{N}\right)^{T} \\ \bar{A}_{v} \mathcal{P}+\bar{B}_{v} \mathcal{N} & -\mathcal{P}\end{array}\right] \prec 0$

for $v=1, \ldots, n_{v}$, which represent underlying matrix vertices, and

$\begin{aligned} & \bar{A}_{v}= {\left[\begin{array}{cccccccc}A_{d v} & 0 & \cdots & 0 & I & 0 & \cdots & 0 \\ -C_{d} A_{d v} & 0 & \cdots & 0 & I & 0 & \cdots & 0 \\ \vdots & \vdots & \ddots & \vdots & \vdots & \vdots & \ddots & \vdots \\ -C_{d} A_{d v} & 0 & \cdots & 0 & I & 0 & \cdots & 0\end{array}\right], } \\ & \bar{B}_{v}=\left[\begin{array}{cccc}B_{d v} & \cdots & B_{d v} \\ -C_{d} B_{d v} & \cdots & -C_{d} B_{d v} \\ \vdots & \ddots & \vdots \\ -C_{d} B_{d v} & \cdots & -C_{d} B_{d v}\end{array}\right],\end{aligned}$

where the matrices $\mathcal{P}$ and $\mathcal{N}$ are given by (17).

If the LMI of (32) holds, stabilizing matrices in the control law (11) are given by (18). 
Table 1

\begin{tabular}{lllll}
\hline Parameters. & & & \\
\hline Sym. & Description & SI value & SI unit & Per unit \\
\hline$J$ & Total inertia & $0.47 \times 10^{-4}$ & $\mathrm{~kg} \mathrm{~m}^{2}$ & 0.0267 \\
$V_{B}$ & Viscous coeff. & $1.1 \times 10^{-4}$ & $\mathrm{~N} \mathrm{~m} \mathrm{~s}$ & 0.0625 \\
$L_{d}$ & $d$-axis inductance & $7.0 \times 10^{-3}$ & $\mathrm{H}$ & 0.4120 \\
$L_{q}$ & $q$-axis inductance & $7.0 \times 10^{-3}$ & $\mathrm{H}$ & 0.4120 \\
$R_{S}$ & Resistance & 2.98 & $\mathrm{Ohm}$ & 0.2781 \\
$\phi_{m g}$ & Flux linkage due to permanent magnet & 0.125 & $\mathrm{~Wb}$ & 0.9102 \\
$i_{\text {rated }}$ & Nominal current & 2.9 & $\mathrm{~A}$ & 0.36 \\
$n_{p}$ & No. of pole pairs & 2 & & \\
\hline
\end{tabular}

Proof. The uncertain process is stable along the pass if every 'vertex' system has this property. The proof now follows identical steps to that of Theorem 1 for each matrix vertex.

In this result as the uncertainty bound increases the feasibility of the LMI (32) could be restricted. Even if LMIs are feasible and control law matrices obtained, slow convergence or excessive control demands could result, as in robust control of other systems. Theorem 2 uses a single Lyapunov function for all polytopes and hence there could be conservativeness associated with the control law design. To reduce the effects of this and potentially augment the uncertainty bounds, a parameter dependent Lyapunov function approach can be used and this is the subject of ongoing research. The results in this paper transfer to norm bounded uncertainty with routine changes and hence the details are omitted.

As in Theorem 1, the control law matrices can be parameterized using the optimization procedure

$\operatorname{maximize}\left(\sum_{i=-w_{l}}^{w_{h}} \operatorname{tr}\left(Q_{i}\right)+\sum_{i=-w_{l}}^{w_{h}} \operatorname{se}\left(N_{i}\right)\right)$

subject to:

$\left[\begin{array}{cc}-\mathcal{P} & \left(\bar{A}_{v} \mathcal{P}+\bar{B}_{v} \mathcal{N}\right)^{T} \\ \bar{A}_{v} \mathcal{P}+\bar{B}_{v} \mathcal{N} & -\mathcal{P}\end{array}\right] \prec 0$,

$\zeta \operatorname{tr}\left(Q_{i}\right) \leq \operatorname{se}\left(N_{i}\right), \quad i=-w_{l}, \ldots, w_{h}$.

For implementation, the control law for either form of uncertainty is

$$
\begin{aligned}
u_{k}(p)= & u_{k-1}(p)+K\left(x_{k}(p)-x_{k-1}(p)\right) \\
& +\sum_{i=-w_{l}}^{w_{h}} K_{i}\left(y_{\text {ref }}(p+i+1)-y_{k-1}(p+i+1)\right) .
\end{aligned}
$$

This control law includes the commonly used phase-lead noncausal ILC law $u_{k}(p)=u_{k-1}(p)+K_{1}\left(y_{\text {ref }}(p+1)-y_{k-1}(p+1)\right)$ as a special case. In general, a moving window of previous trial output information together with the state actuated second term is used to compute the next trial input. If all entries in the state vector are not available for measurement, the results in this paper generalize to a control law of the form (35) where the term $K\left(x_{k}(p)-x_{k-1}(p)\right)$ is replaced by $\widetilde{K}\left(y_{k}(p)-y_{k-1}(p)\right)$ but a preliminary stabilizing control loop may be required. This form of output only control law without the moving window has been experimentally verified [9]. The new feature of the moving window has the dual purpose of reducing conservativeness and improving performance. The static control law using output only information is, in general, weaker that its state vector based alternative and the resulting LMIs can be more conservative.

The window can be symmetrical around the reference point $k$ or non-symmetrical; also its length can be adjusted in accordance with needs of a particular application, where the wider the window the greater the numerical cost. Moreover, it is not possible to develop formulas for determining the selection of the moving window parameters $w_{l}$ and $w_{h}$ and instead these must be decided on a case-by-case basis. The choice of the parameter $w_{h}$ determines how the noncausal data is included in the control law and, given that there is no formula for any currently available ILC design using noncausal data, can be varied in simulation to determine the value appropriate for a given application. One other possible benefit from the choice of the parameters $w_{l}$ and $w_{h}$ is to obtain a control law with less conservative LMIs and acceptable performance. It is also possible to modify this approach to the "sparse" case when not all points within $\left\{-w_{l}, w_{h}\right\}$ are used. The design developed in this paper provides an alternative to the frequency domain design in [5] and its relative merits are discussed again in the conclusion section. Next a case study is given.

\section{Case study}

In this section the ILC design of the previous sections is applied to the model of an electric motor system, similar to that used in repetitive control studies [12], with dynamics described, with disturbances assumed negligible, by

$$
\begin{aligned}
& \frac{d \omega_{m}(t)}{d t}=\frac{1}{J}\left(\frac{3}{2} n_{p} \phi_{m g} i_{q}(t)-V_{B} \omega_{m}(t)\right), \\
& i_{q}(t)=-\frac{1}{\gamma} i_{q}(t)+\frac{1}{\gamma} i_{q}^{*}(t), \\
& \dot{\theta(t)}=\omega_{m}(t) \cdot\left(\frac{\omega_{b}}{2 \pi}\right),
\end{aligned}
$$

where $\omega_{b}=315.315(\mathrm{rad} / \mathrm{s}), \gamma=6.8 \times 10^{-4}, i_{q}^{*}(t)$ is the input, $\theta(t)$ is the output and $T_{L}(t)$ is the disturbance acting on the system. Per unit parameters are used and are given in Table 1.

Introducing the state vector, input and output

$x(t)=\left[\begin{array}{c}\omega_{m}(t) \\ i_{q}(t) \\ \theta(t)\end{array}\right], \quad u(t)=i_{q}^{*}(t), y(t)=\theta(t)$,

gives the system state-space model

$\dot{x}(t)=A_{c} x(t)+B_{c} u(t)$,

$y(t)=C_{c} x(t)$,

where

$A_{c}=\left[\begin{array}{ccc}-\frac{V_{B}}{J} & \frac{3 n_{p} \phi_{m g}}{2 J} & 0 \\ 0 & -\frac{1}{\gamma} & 0 \\ \frac{\omega_{b}}{2 \gamma} & 0 & 0\end{array}\right]$,

$B_{c}=\left[\begin{array}{c}0 \\ \frac{1}{\gamma} \\ 0\end{array}\right], \quad C_{c}=\left[\begin{array}{lll}0 & 0 & 1\end{array}\right]$. 


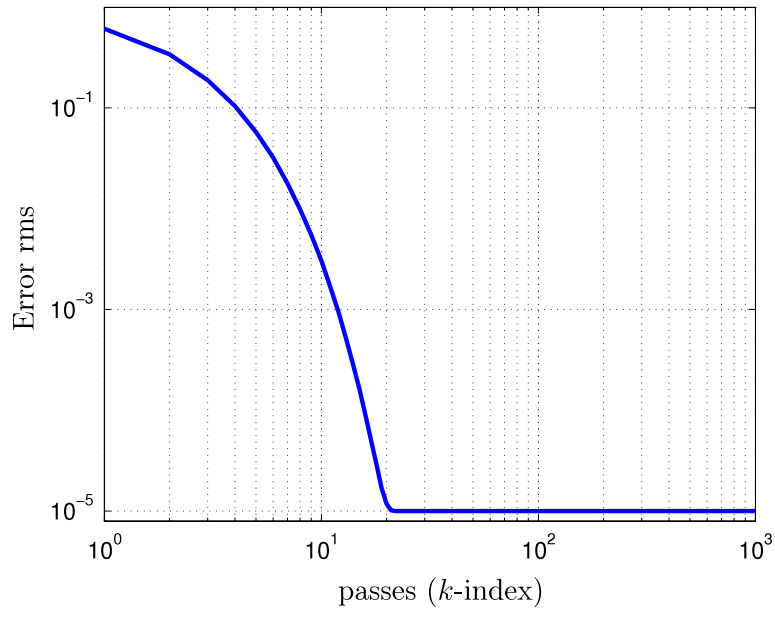

Fig. 1. The root mean square error for the nominal model design where the very small non-zero values after $k=20$ are due to numerical approximations.

For control law design the state-space dynamics have been sampled at $T_{s}=200 \mu \mathrm{s}$, the pass length is $2 \mathrm{~s}$ and the reference signal is

$y_{\text {ref }}(t)=\sin (\pi t)+0.5 \sin (2 \pi t)+0.5 \sin (3 \pi t)$

with boundary conditions

$x_{0}(p)=0, \quad u_{0}(p)=0, \quad 0 \leq p \leq \alpha-1$,

$x_{k}(p)=0, \quad u_{k}(p)=0, \quad e_{k}(p)=0, \quad y_{k}(p)=0$,

$p \in\left\{-w_{l}, \ldots,-1\right\} \cup\left\{\alpha, \ldots, \alpha+w_{h}-1\right\}$,

where $\alpha=10,000$ and $w_{l}=w_{h}=1$.

For the following motor parameters in Table 1

$V_{B}=0.0625, \quad J=0.0267$,

$\phi_{m g}=0.9102, \quad \omega_{b}=315.315$

the optimization procedure (26a)-(26c) gives the control law matrices for the nominal model based design with $\zeta=2.1$.

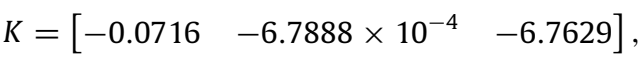

$K_{-1}=K_{1}=2.2065, \quad K_{0}=6.1011$.

Pass-to-pass error convergence is measured by the root mean square error

$e_{\mathrm{rms}}(k)=\sqrt{\frac{1}{\alpha} \sum_{p=0}^{\alpha-1} e_{k}(p)^{T} e_{k}(p)}$.

Fig. 1 shows $e_{\mathrm{rms}}(k)$ for this design and Fig. 2 the output on the first six passes, which confirms that tracking of the reference occurs after a few passes.

In any motor, the $\phi_{m g}$ parameter can vary and to assess the robust design it is assumed that

$\phi_{m g} \in[0.6,0.95]$.

This gives a polytopic uncertainty with two vertices given by the matrices

vertex 1:

$A_{d 1}=\left[\begin{array}{ccc}0.9995 & 0.0117 & 0 \\ 0 & 0.7452 & 0 \\ 0.01 & 6.1481 \times 10^{-5} & 1\end{array}\right]$,

$B_{d 1}=\left[\begin{array}{c}17.174 \\ 1.0959 \times 10^{3} \\ 0.0904\end{array}\right]$,

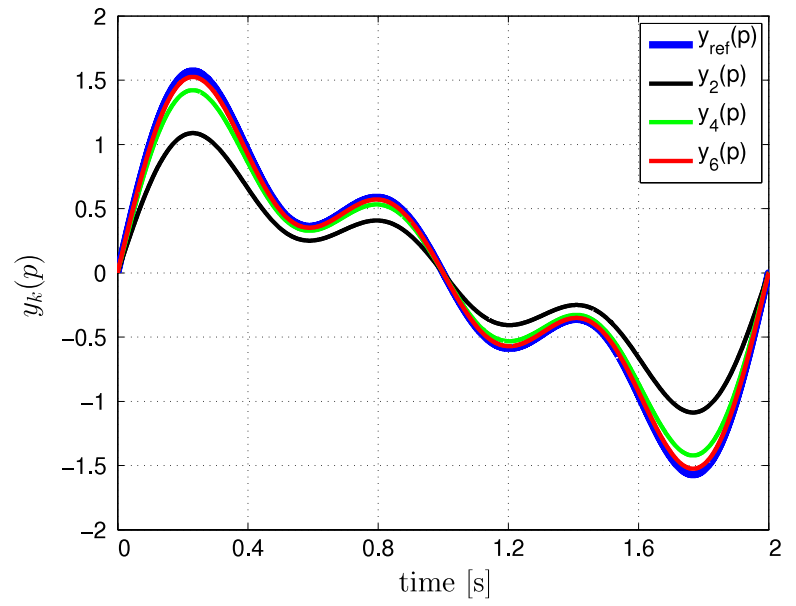

Fig. 2. The output on passes $k=2,4$ and 6 compared with the reference signal for the nominal model design.

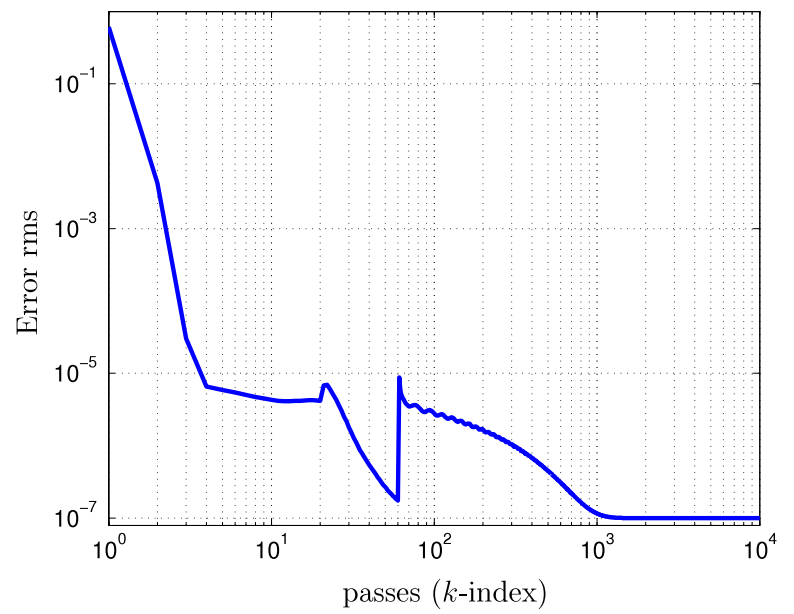

Fig. 3. The root mean square error for the robust design with the value of $\phi_{m g}$ switched at $k=20$ and $k=60$.

vertex 2:

$A_{d 2}=\left[\begin{array}{ccc}0.9995 & 0.0185 & 0 \\ 0 & 0.7452 & 0 \\ 0.01 & 9.7344 \times 10^{-5} & 1\end{array}\right]$,

$B_{d 2}=\left[\begin{array}{c}27.192 \\ 1.0959 \times 10^{3} \\ 0.1432\end{array}\right]$,

and the nominal model $\phi_{m g}=0.9102$ is within the considered polytope.

The optimization procedure (34a)-(34c) with $\zeta=1.1$ gives following control law matrices for this case:

$K=\left[\begin{array}{lll}-0.066 & -6.8027 \times 10^{-4} & -4.8474\end{array}\right]$,

$K_{-1}=K_{1}=1.0504, \quad K_{0}=2.78$.

The controlled system has been simulated with $\phi_{m g}=0.6$, until $k=20$ when the value of this parameter was switched to $\phi_{m g}=$ 0.95 and finally at $k=60$ was switched back to its starting value, resulting in Figs. 3-5. As expected, the pass error increases stepwise each time the value of $\phi_{m g}$ changes but is quickly reduced over the next few passes.

Fig. 4 shows the reference and output signals for passes $k=$ 19, 20 and 21, i.e, before, on and after the first switch and Fig. 5 likewise for the second switch. In both cases, only a segment of the along the pass dynamics is shown for clarity. These confirm 


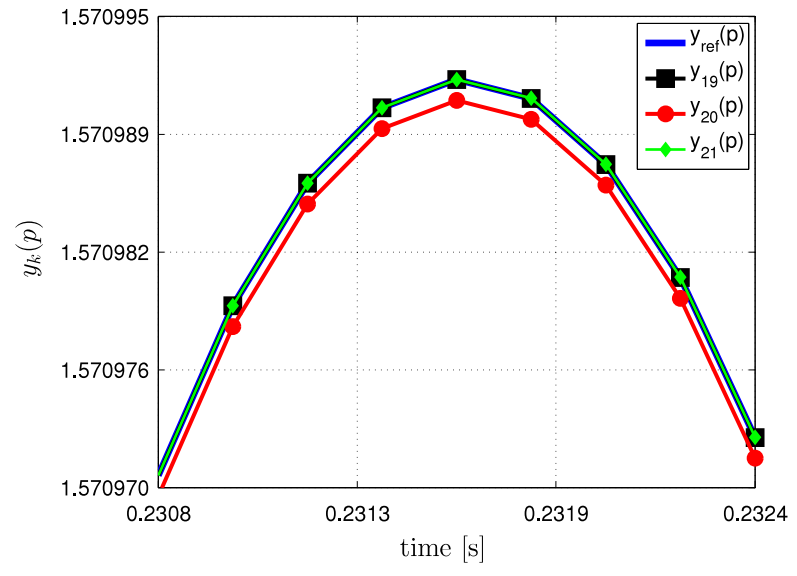

Fig. 4. A segment of the output on passes $k=19,20$ and 21 compared with the reference signal for the robust design just before and after the first switch in $\phi_{m g}$.

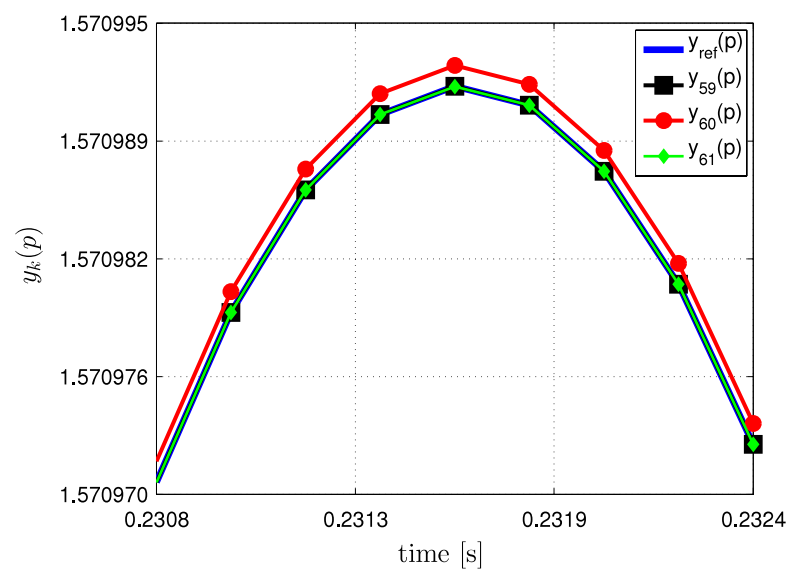

Fig. 5. A segment of the output on passes $k=59,60$ and 61 compared with the reference signal for the robust design just before and after the second switch in $\phi_{m g}$.

that there is an error induced on the pass where the switch occurs but is damped out on the next trial. This demonstrates that the new ILC law is robustly stable along the pass and can ensure fast pass-to-pass error convergence. The switch between $\phi_{m g}=0.6$ and $\phi_{m g}=0.95$ is maximum realistic with physical motors of the form considered and for this uncertainty bound the design is feasible. This provides supporting evidence as to the viability of this design in applications and motivates further development to provide design support for users.

To illustrate the use of noncausal data, a comparison with the robust control law of (10) has been undertaken. Applying a simplified version of Theorem 3 in [8] gives the following control law matrices:

$K_{1}=\left[\begin{array}{lll}-0.0629 & -6.8003 \times 10^{-4} & -3.8375\end{array}\right]$,

$K_{2}=1.4319$.

Fig. 6 shows the root mean error for this last control law and in comparison to Fig. 3 its convergence is much slower. Simulations also show better relative performance for this new design just before and after the switches in $\phi_{m g}$.

\section{Conclusions}

This paper has developed new results on ILC design in the repetitive process setting, with particular emphasis on the inclusion of noncausal previous pass data in the control law and robustness. The main result is an LMI based design whose performance has been assessed in simulation on a motor model from the literature,

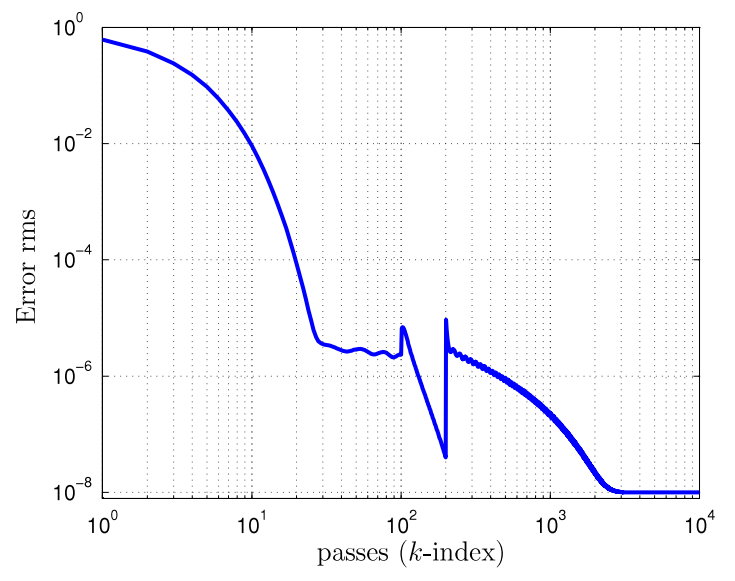

Fig. 6. The root mean square error for the robust control law design in [9].

demonstrating that the use of previous trial data in ILC can make a significant difference to the performance achieved. Areas for further development include design with disturbance rejection, performance bounds and trade-offs, for which an $\mathscr{H}_{\infty}$ setting is a starting point where [7] contains basic results for similar repetitive process problems. As in all other control design algorithms there are parameters that must be chosen based on the knowledge of the application and this is the case for $w_{l}$ and $w_{h}$ in the control law (35).

The analysis and case study in this paper have established that the use of noncausal previous trial data beyond a single phase-lead term has merits in ILC design and provides the results necessary for design to begin, i.e., pass-to-pass error convergence and control law design formulas with an extension to robustness. In the latter aspect, the repetitive process setting does not, in comparison to the lifting design, encounter products of matrices defining the uncertainty. A detailed comparison with the lifted approach and other design alternatives can only be attempted after significant further development for which some areas for possible investigation have also been given.

\section{References}

[1] S. Arimoto, S. Kawamura, F. Miyazaki, Bettering operation of robots by learning, J. Robot. Syst. 1 (2) (1984) 123-140.

[2] D.A. Bristow, M. Tharayil, A. Alleyne, A survey of iterative learning control, IEEE Control Syst. Mag. 26 (3) (2006) 96-114.

[3] H.-S. Ahn, Y.-Q. Chen, K.L. Moore, Iterative learning control: brief survey and categorization, IEEE Trans. Syst. Man Cybern. C: Appl. Rev. 37 (6) (2007) 1099-1121.

[4] C.T. Freeman, E. Rogers, A. Hughes, J.H. Burridge, K.L. Meadmore, Iterative learning control in health care: electrical stimulation and robotic-assisted upper-limb stroke rehabilitation, IEEE Control Syst. 32 (1) (2012) 18-43.

[5] J.J.M. van de Wijdeven, M.C.F. Donkers, O.H. Bosgra, Iterative learning control for uncertain systems: noncausal finite time interval robust control design, Internat. J. Robust Nonlinear Control 21 (14) (2011) 1645-1666.

[6] J.E. Kurek, M.B. Zaremba, Iterative learning control synthesis based on 2D system theory, IEEE Trans. Automat. Control 38 (1) (1993) 121-125.

[7] E. Rogers, K. Gałkowski, D.H. Owens, Control Systems Theory and Applications for Linear Repetitive Processes, in: Lecture Notes in Control and Information Sciences, vol. 349, Springer-Verlag, Berlin Heidelberg, 2007.

[8] Ł Hładowski, K. Gałkowski, Z. Cai, E. Rogers, C.T. Freeman, P.L. Lewin, Experimentally supported 2D systems based iterative learning control law design for error convergence and performance, Control Eng. Pract. 18 (4) (2010) 339-348.

[9] Ł Hładowski, K. Gałkowski, Z. Cai, E. Rogers, C.T. Freeman, P.L. Lewin, Output information based iterative learning control law design with experimental verification, ASME J. Dyn. Syst. Meas. Control 134 (2) (2012) 021012/1-021012/10.

[10] R.P. Roesser, A discrete state-space model for linear image processing, IEEE Trans. Automat. Control 20 (1) (1975) 1-10.

[11] K. Gałkowski, B. Cichy, E. Rogers, J. Lam, Stabilization of a class of uncertain wave discrete linear repetitive processes, in: Proceedings of the 45th IEEE Conference on Decision and Control, San Diego, USA, 2006, pp. 1435-1440.

[12] L. Zhou, J. She, M. Wu, J. Zhang, Design of a robust modified repetitive-control system for a periodic plant, ASME J. Dyn. Syst. Meas. Control 134 (1) (2012) 011023/1-011023/7. 